\title{
Clustering of Pentacene and Functionalized Pentacene Ions in a Matrix-Assisted Laser Desorption/Ionization Orthogonal TOF Mass Spectrometer
}

\author{
Svitlana V. Shcherbyna and Diethard K. Bohme \\ Department of Physics and Astronomy, Department of Chemistry, Center for Research \\ in Mass Spectrometry, York University, Toronto, Ontario, Canada \\ Vladimir I. Baranov and Alexander Loboda \\ MDS Sciex, Concord, Ontario, Canada \\ Christopher R. Swartz and John E. Anthony \\ Department of Chemistry, University of Kentucky, Lexington, Kentucky, USA
}

\begin{abstract}
A high-performance orthogonal time-of flight (TOF) mass spectrometer, in combination with the matrix assisted laser desorption/ionization (MALDI) source operating at elevated pressure $(\sim 1$ torr in $\mathrm{N}_{2}$ ), was used to perform MALDI-TOF analyses of pentacene and some of its derivatives with and without an added matrix. These molecules are among the most interesting semiconductor materials for organic thin film transistor applications (OTFT). The observation of ion-molecule reactions between "cold" analyte ions and neutral analyte molecules in the gas phase has provided some insight into the mechanism of pentacene cluster formation and its functionalized derivatives. Furthermore, some of the matrices employed to assist the desorption/ionization process of these compounds were observed to influence the outcome via ion-molecule reactions of analyte ions and matrix molecules in the gas phase. The stability and reactivity of the compounds and their clusters in the MALDI plume during gas-phase expansion were evaluated; possible structures of the resulting clusters are discussed. The MALDI-TOF technique was also helpful in distinguishing between two isomeric forms of bis-[(triisopropylsilyl)-ethynyl]-pentacene. (J Am Soc Mass Spectrom 2006, 17, 222-229) (c) 2006 American Society for Mass Spectrometry
\end{abstract}

$\mathrm{P}$ entacene and its functionalized derivatives are among the most interesting semi-conducting materials for use in organic thin film transistors (OTFT). This material is known to have the highest mobility of charged carriers among thin-film organic semiconductors [1-3].

Molecular order is a significant factor in the performance of devices based on organic semiconductors. Recent studies have demonstrated that targeted modifications that increase orbital overlap in the solid-state can improve the conductivity by more than an order of magnitude [1]. For example, targeted functionalization of pentacene has been shown to be an effective modifier of the electronic properties of the resulting crystals and thin films [1, 2], inducing self-assembly of the aromatic moieties into $\pi$-stacked arrays to enhance intermolecular orbital overlap. Unlike pentacene, functionalized pentacene is soluble in some organic solvents and can be

Published online January 18, 2006

Address reprint requests to Dr. D. K. Bohme, Department of Chemistry, York University, 4700 Keele Street, Toronto, Ontario M3J 1P3, Canada. E-mail: dkbohme@yorku.ca spin-cast (a readily available technique for thin film preparation) for potential application studies. Intensive experimental studies have been performed in this area of research [3, 4].

In this work, we present a mass spectrometric study of pentacene and four derivatives: symmetric 6,13bis-[(triisopropylsilyl)-ethynyl]-pentacene (TIPS), 5,14bis-[(triisopropylsilyl)-ethynyl]-pentacene (offset TIPS or o-TIPS), 6,13-bis-[(triisopropylsilyl)-ethynyl]-pentacene-2,3-dicarbonitrile or TIPS $(\mathrm{CN})_{2}$, and 6,13-bis[(triisopropylsilyl)-ethynyl]-pentacene-2,3,9,10-tetracarbonitrile or TIPS $(\mathrm{CN})_{4}$ (derivatives with additional cyanide groups attached to the pentacene) (see Figure 1). The first two selected funtionalized pentacene derivatives have demonstrated significant conductivity enhancement [2]; the carbonitrile derivatives are newly synthesized molecules and not yet examined as material for OTFTs.

Because most OTFT devices are prepared by vacuum sublimation, any experiment that helps us to understand the nature of cluster formation of these organic semiconductors can lead to a better understanding of how the films are grown. For example, the tendency of 
a)

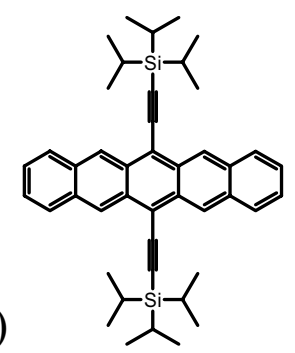

c)

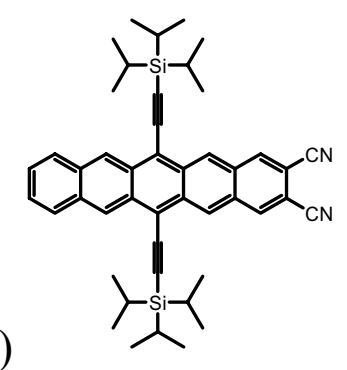

b)

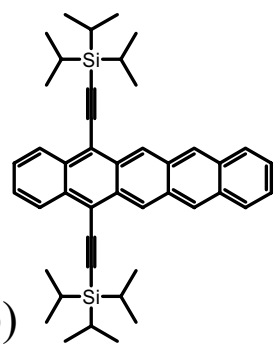

d)

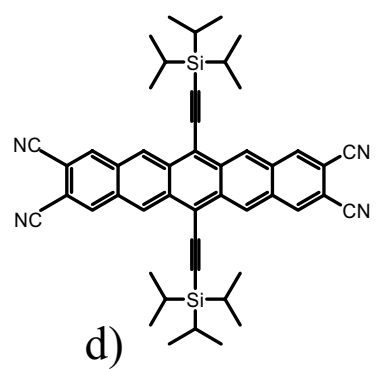

Figure 1. Two-dimensional structures for (a) 6,13-bis(triisopropylsilylethynyl) pentacene or TIPS; (b) 5,14-bis(triisopropylsilylethynyl) pentacene or o-TIPS; (c) 2,3-dicyano-6,13-bis(triisopropylsilylethynyl) pentacene or TIPS(CN $)_{2}$; (d) 2,3,9,10-tetracyano-6,13bis(triisopropylsilylethynyl) pentacene or $\operatorname{TIPS}(\mathrm{CN})_{4}$.

pentacene and its derivatives to aggregate in the gas phase might provide insight into their tendency to form highly crystalline films by vapor deposition. If the aggregates are large enough to serve as nucleation sites, then perhaps this is what leads to the large crystalline grain sizes that are critical to the device performance.

The mass-spectrometric investigations reported here were performed with a novel matrix-assisted desorption/ionization (MALDI) technique at elevated pressures [5]. In the application of this MALDI technique, the sample is usually premixed with a highly absorbing matrix compound that transforms the laser energy into excitation/ionization energy for the sample molecules. A fine balance has to be achieved between sufficient energy transfer and suppression of unimolecular decomposition. The functionalized pentacene samples were deposited and analyzed with and without premixing with the matrix. The selected matrices were TCNQ, $\mathrm{DHB}$, and $\alpha$-CHC. As will be discussed later, the latter two matrices were not suitable because of chemical reactions of matrix molecules with ions and molecules of the analyte in the laser-induced plume.

Under experimental conditions described in [5], we observe that some of the analytes under consideration demonstrate different degrees of clustering (adduct formation). Recent investigations [6] of the mechanism of matrix adduct formation in MALDI conducted at elevated pressures have confirmed that adducts (clusters) between ionized and neutral molecules develop in the dense plume of matrix molecules rather than by desorption of ionized matrix clusters. Due to gas dynamic expansion of the plume, the analyte ions undergo multiple collisions with neutral molecules and cool down under adiabatic conditions. Formation of the ionic clusters is usually exothermic and is electrostatic in nature. This suggests an anti-Arrhenius dependence of the rate constants for cluster formation on temperature. An increase of ion-molecule reaction rate with decreasing temperature is quite common for exothermic association reactions being a consequence of the increased lifetime of the intermediate collision complex. Additional collisional cooling by a buffer gas in the RF only ion guide under very low RF heating conditions also reduces the monomolecular decay ("evaporation" of neutral molecules) of clusters before analysis.

\section{Experimental}

Pentacene was purchased from (Sigma) Aldrich (Oakville, Canada). TIPS, o-TIPS, TIPS(CN) $)_{2}$, and TIP$\mathrm{S}(\mathrm{CN})_{4}$ were synthesized in the Synthetic Organic and Materials Chemistry Laboratory at the University of Kentucky. 6,13-bis(triisopropylsilylethynyl) pentacene (TIPS) and 5,14-bis(triisopropylsilylethynyl) pentacene (o-TIPS) were prepared as described elsewhere [1]. 2,3-Dicyano-6,13-bis(triisopropylsilylethynyl) pentacene, TIPS $(\mathrm{CN})_{2}$, and 2,3,9,10-tetracyano-6,13-bis(triisopropylsilylethynyl) pentacene (TIPS $(\mathrm{CN})_{4}$ ) were prepared by the palladium-catalyzed cyanation [7] of the corresponding dibromo- and tetrabromo-pentacene derivatives [8]. All compounds were purified by repeated crystallization from acetone and then hexanes. Toluene $(98.8 \%)$ and methanol (100\%) used for solutions were purchased from Sigma-Aldrich. Stock solutions were prepared with concentrations of $940 \mathrm{nmol} \mathrm{ml}^{-1}$ (TIPS, o-TIPS), $871 \mathrm{nmol} \mathrm{ml}^{-1}$ (TIPS(CN) $)_{2}$ ), and $812 \mathrm{nmol} \mathrm{ml}^{-1}$ $\left(\mathrm{TIPS}(\mathrm{CN})_{4}\right)$ in toluene/methanol 3:7 (vol/vol). The toluene and methanol were degassed ultrasonically before use.

The MALDI matrix 7,7,8,8-tetracyanoquinodimethane (TCNQ) was obtained from Sigma-Aldrich (98\%). 2,5-Dihydroxybenzoic acid (DHB) and $\alpha$-cyano-4-hydroxynnamic acid $(\alpha-\mathrm{CHC})$ were purchased as matrix solutions from Agilent Technologies (Palo Alto, CA).

All experiments were performed with the MALDITOF instrument prOTOF 2000 (Perkin Elmer SCIEX, Concord, Canada). A similar instrument recently has been described in detail [5]. Briefly, the instrument contains a MALDI source pressurized by a buffer gas $\left(\mathrm{N}_{2}\right)$ up to ca. 1 torr (the gas flow in the target region was $200 \pm 40 \mathrm{sccm}$ ), followed by a sampling cone, an RF only quadrupole ion guide, and an orthogonal injection time-of-flight (TOF) mass spectrometer. The collisionalfocusing ion guide decouples the ion production from the TOF measurement. Ions become successfully thermalized as they experience numerous collisions while traversing the ion guide. Collisions also reduce the internal energy of the ions and virtually eliminate the monomolecular fragmentation of an analyte.

An optical fibre with a core diameter $200 \mu \mathrm{m}$ (InnovaQuartz, Phoenix, AZ) delivers pulsed laser light to the MALDI target plate to ensure spatially homoge- 
neous energy deposition. The laser light was generated by a nitrogen laser (337ND-Sx: Spectra-Physics). The accelerating voltage in the TOF section was set to $16 \mathrm{kV}$ to ensure efficient detection of MALDI-generated ions.

All samples, except pentacene, were deposited onto the MALDI target plate using the nebulizer assistedcontinuous track technique. This sample preparation technique was utilized for both the MALDI and LDI experiments. During deposition, the target plate was placed on top of the syringe pump (Harvard Apparatus, Holliston, MA) and moved at a constant speed of 30 $\mathrm{mm} \mathrm{min}^{-1}$. An electrospray needle with an i.d. of 125 $\mu \mathrm{m}$ was used to nebulize the stock solution with or without matrix (no electric potential was applied). Compressed air was passed through a concentric tube to assist in the nebulization. The solution was delivered with a second syringe pump (Harvard Apparatus) at 2 $\mathrm{L} \min ^{-1}$. In the experiments with matrix/analyte mixtures the stock solutions were diluted down to $0.001 \%$ and then mixed with matrix in proportion matrix/ analyte $3 / 1$ (vol/vol). The target deposition rate was $0.25 \mathrm{ml} \mathrm{min}^{-1}$. A similar set up that has been developed and used previously for MALDI film deposition has been described elsewhere [9].

Nebulizer-assisted deposition is relatively easy to implement, forms a continuous track that is well suited for MALDI sampling, and offers near steady-state desorption conditions. Furthermore, the continuous track increases the efficiency of multiple laser ablations. This deposition process offers an advantage over wet spotting by producing a more controlled and reproducible analyte/matrix cocrystallization, by decreasing the dependence on surface quality and history, and by allowing better control over track thickness $[9,10]$.

Nebulizer-assisted deposition cannot be applied to pentacene since, unlike functionalized derivatives, it is not easily soluble in most organic solvents. Therefore, pentacene powder was dispersed in toluene in an ultrasonic bath and then deposited by the wet spotting method into the MALDI target.

\section{Results}

\section{Evaluation of MALDI Matrices}

In our investigation of pentacene derivatives, advantages of the TCNQ matrix are self-evident and it is the best among tested matrices for analytical purposes. TCNQ has proven to be the most suitable matrix for the characterization of polycyclic aromatic hydrocarbons (PAHs) with MALDI mass spectrometry. Recent studies have shown that this new matrix exhibits some interesting properties that can be useful for the characterization of compounds that tend to fragment during the MALDI process [11]. For example, TCNQ decreases the fragmentation of analyte due to its low desorption threshold. Because of its ability to accept electrons, this matrix promotes the formation of analyte radical cations and thus is well adapted for the characterization of

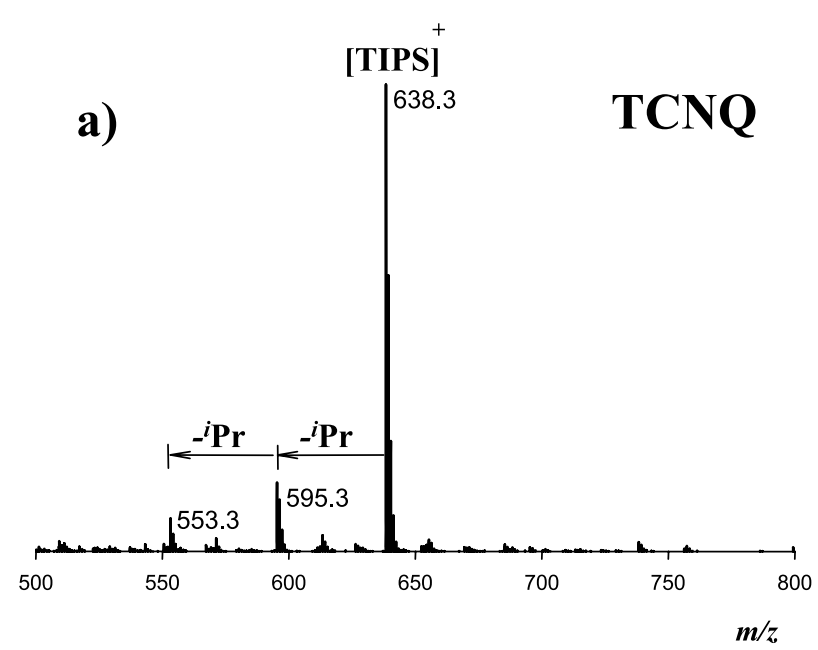

b)

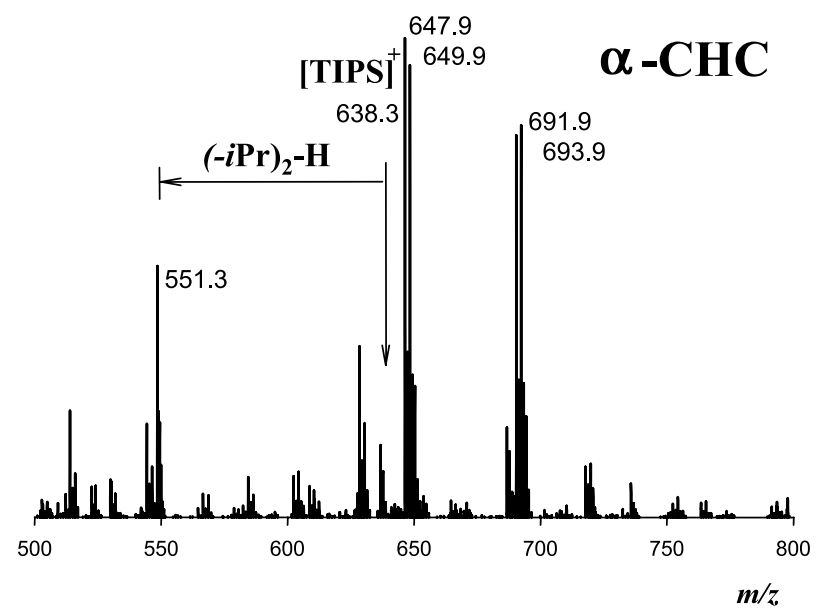

Figure 2. Comparison of MALDI mass spectra recorded for TIPS using TCNQ (a) and $\alpha$-CHC (b) matrices. The laser fluence was set at 60 relative units. (The intensity axis has a linear scale).

analytes absorbing at $337 \mathrm{~nm}$ (MALDI $\mathrm{N}_{2}$ laser wavelength).

When TCNQ was employed as a matrix, the analytes were present in the spectra as radical cations presumably produced by electron detachment. No protonation was detected and no clusters of primary ions and neutral matrix molecules were observed. In the case of pentacene, the pentacene ion $(\mathrm{m} / \mathrm{z} 278.1)$, the TCNQ matrix ion $(\mathrm{m} / \mathrm{z} 204.1)$, and an ion at $\mathrm{m} / \mathrm{z}$ 368.4 , possibly a product of an analyte/matrix reaction, were reproducibly observed. The TIPS spectra have included the primary ion $(\mathrm{m} / \mathrm{z} 638.3)$, the matrix ion $(m / z 204.1)$, and some fragmentation product ions at $m / z 595.3,553.3$, likely due to loss of one and two isopropyl groups, respectively (see Figure 2a). o-TIPS has a similar spectrum as TIPS except for a prominent $\mathrm{m} / \mathrm{z} 818.5$ signal. This is the unique feature of the spectrum of the o-TIPS isomer and a possible explanation is presented below. TIPS $(\mathrm{CN})_{2}$ has an intensive primary ion signal at $\mathrm{m} / \mathrm{z} 688.4$ as well as a unique product ion at $\mathrm{m} / z$ 923.4. $\operatorname{TIPS}(\mathrm{CN})_{4}$ has the 
weakest primary ion signal in comparison with other compounds. Its primary ion $(\mathrm{m} / \mathrm{z} 738.3)$ was suppressed by extensive fragmentation and products of multiple matrix-analyte ion-molecule reactions (for example, the strong $\mathrm{m} / \mathrm{z} 973$ signal).

The DHB and $\alpha$-CHC matrices used here for UVMALDI are well studied for a wide range of analytes [12]. When DHB and $\alpha-\mathrm{CHC}$ were used as matrices in our study, the primary ion signals of TIPS and o-TIPS were strongly suppressed (as in Figure $2 \mathrm{~b}$ obtained with an $\alpha$-CHC matrix compared with the TIPS/TCNQ spectrum in Figure 2a).

Some analyte ion fragments are observed in the spectrum with $\alpha$-CHC matrix as well as the appearance of large "doublet" peaks at $m / z=647.9$ and 649.9 and $m / z=691.9$ and 693.9. The peak at $m / z 691.9$ may be an adduct of fragmented TIPS (with complete loss of one of the functional groups), one molecule of $\alpha-\mathrm{CHC}$, and an additional $\mathrm{COOH}$ group from the matrix. Also the peak at $m / z 647.9$ could be the same adduct that has lost $\mathrm{CO}_{2}$. The difference of $2 \mathrm{u}$ between $\mathrm{m} / \mathrm{z} 647.9$ and 649.9 and between 691.9 and 693.9 is probably the result of a substitution of the $\mathrm{CN}$ group in $\alpha$-CHC by CO.

The DHB matrix has a similar effect on TIPS and o-TIPS producing multiple fragments and products of ion-molecule reactions. Moreover, the primary ion signals of $\operatorname{TIPS}(\mathrm{CN})_{2}$ and $\operatorname{TIPS}(\mathrm{CN})_{4}$ were totally suppressed in the presence of DHB and $\alpha$-CHC matrices. Extensive fragmentation (loss of $\mathrm{CN}$ and (tri-isopropylsilyl)-ethynyl groups) as well as clustering of fragments and reaction products with matrix were observed. Perhaps such reactivity should not be surprising: the cinnamic acid has a conjugated double-bond, and these types of functional groups tend to react as dienophiles in Diels-Alder type reactions with acenes. This could happen in the laser-induced plume. Loss of the $\mathrm{CN}$ groups due to this reaction is not likely; however, it can be attributed to thermal fragmentation. The dihydroxybenzoic acid might react with the cyano containing compounds by protonation. In this case, one should expect partial loss or displacement of the $\mathrm{CN}$ group.

Besides the chemical reactivity of the MALDI matrix, three other parameters influence ion production in the MALDI source: the laser fluence, the pressure in the ionization region, and the declustering potential (DP). Unfortunately, the prOTOF 2000 software offers laser fluence in relative units. The laser fluence of 60 relative units was fixed for all experiments presented here. The pressure in the ionization region was 1 torr of nitrogen. The declustering potential as well could be called the drift potential between target and cone [5]. In an attempt to minimize the ion energy and reduce friction with the buffer gas, the DP was reduced to $0 \mathrm{~V}$ with a significant drop in the overall ion current. However, the fragmentation and reactivity of analyte ions could not be suppressed significantly, even under very soft ion formation and cooling conditions.

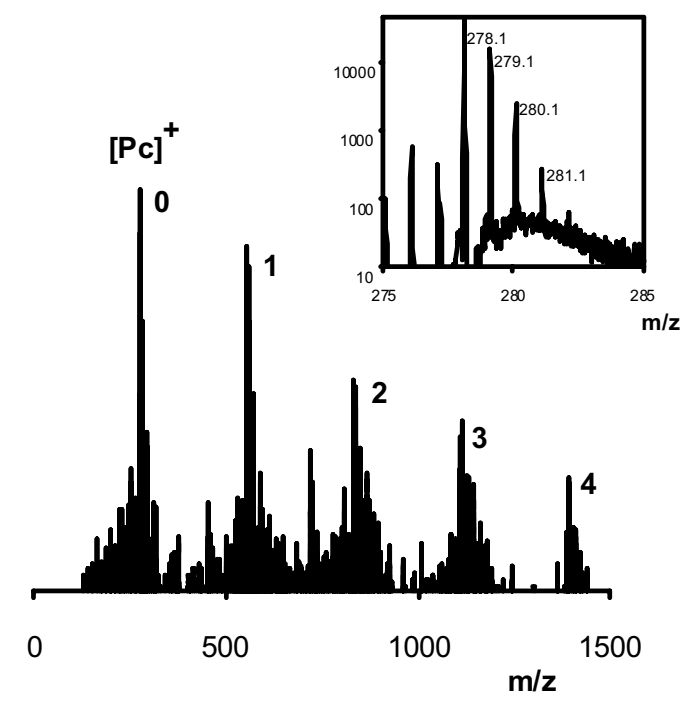

Figure 3. LDI spectrum recorded for pentacene at a source pressure of $\sim 1$ torr with a declustering potential (DP) $=5 \mathrm{~V}$. (The intensity axis has a logarithmic scale). The insert shows the higher-resolution spectra for the monomer ions.

Perhaps not surprisingly, the pentacene derivatives also could be analyzed in the absence of matrix using direct laser desorption ionization (LDI). Under these conditions, self-MALDI prevails and reactions with "foreign" matrix molecules can be avoided.

\section{Laser Desorption Ionization (LDI)}

LDI is generally considered to be a "hard" ionization method, especially in comparison with MALDI, and excessive fragmentation is expected. Without the help of a matrix, a significant number of ions can be produced only above a certain level of laser fluence, which is determined experimentally as an acceptable compromise between fragmentation and analyte ion signal [5].

The LDI spectra obtained for pentacene, TIPS, oTIPS, TIPS $(\mathrm{CN})_{2}$, and TIPS $(\mathrm{CN})_{4}$ are presented in Figures 3,4 , and 5 . The predominant ionization process is electron detachment for all compounds; protonated primary ions were not observed.

Figure 3 illustrates the mass spectrum of pentacene recorded at 1 torr $\mathrm{N}_{2}$ and DP of $5 \mathrm{~V}$. The main spectral features correspond to the primary ionized pentacene and its clusters $\mathrm{Pc}^{+}(\mathrm{Pc})_{\mathrm{n}}$ with $\mathrm{n}$ up to 4 at $\mathrm{m} / \mathrm{z} 556.24$, 833.37, 1112.50, and 1390.0, respectively. The cooling time was estimated to be $\sim 1 \mu \mathrm{s}$ [5] and fragmentation was effectively suppressed. The cluster formation can be suppressed by increasing DP up to $10 \mathrm{~V}$, under which conditions only the first two cluster ions were observed. The mass spectrum of TIPS is shown in Figure 4a and again indicates extensive cluster formation. Cluster ions $\mathrm{TIPS}^{+}(\mathrm{TIPS})_{\mathrm{n}}$ are observed with $\mathrm{n}$ up to 5 at $\mathrm{m} / \mathrm{z}$ 1276.76, 1916.13, 2553.52, 3191.90, and 3830.28 , respectively. It was demonstrated [5] that clusters are formed in the LDI plume, presumably accord- 


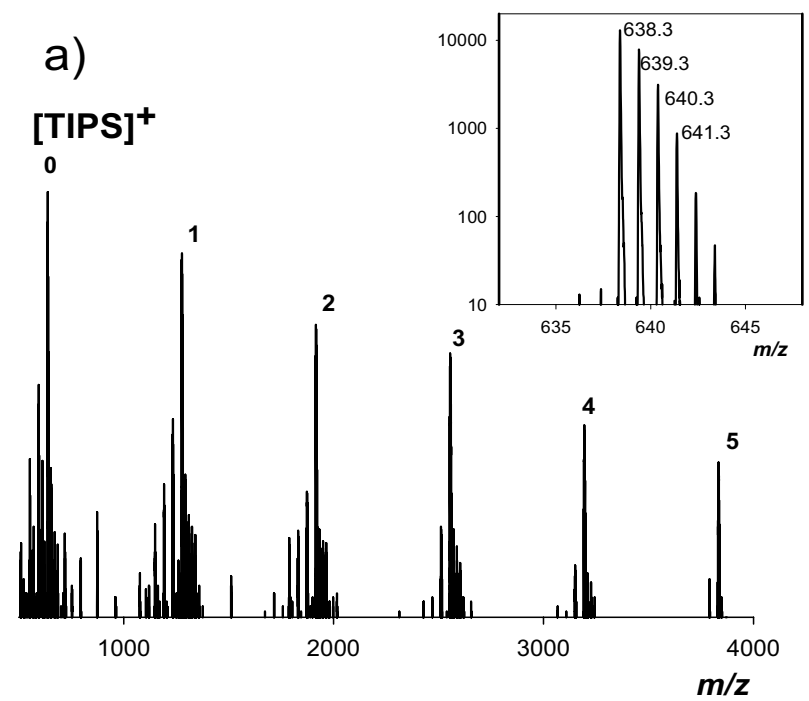

b)

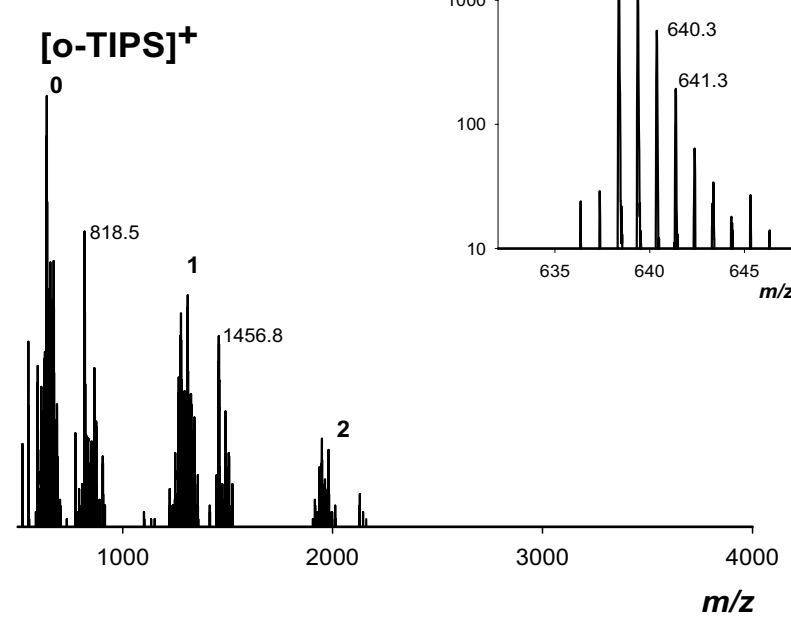

Figure 4. LDI spectra recorded for (a) TIPS and (b) o-TIPS at a source pressure of $\sim 1$ torr with a declustering potential (DP) $=5$ $\mathrm{V}$. (The intensity axis has a logarithmic scale). The inserts show the higher-resolution spectra for the monomer ions.

ing to the following reaction of the analyte cations $\mathrm{M}^{+}$ in the presence of the buffer gas:

$$
\mathrm{M}^{+}(\mathrm{M})_{\mathrm{n}}+\mathrm{M}+\mathrm{N}_{2} \rightarrow \mathrm{M}^{+}(\mathrm{M})_{\mathrm{n}+1}+\mathrm{N}_{2}
$$

In sharp contrast, the spectrum of o-TIPS shown in Figure $4 \mathrm{~b}$ and taken under the same experimental conditions indicates a much lower extent of cluster formation. The dimer cluster ions and only traces of the trimer are evident (note that the ion intensity scale is logarithmic).

Another distinguishable feature of the o-TIPS spectrum is the presence of relatively strong peaks at $\mathrm{m} / \mathrm{z} 818.51, \mathrm{~m} / \mathrm{z}$ 1456.89, and $\mathrm{m} / \mathrm{z}$ 2095.25. We attribute the peak at $\mathrm{m} / \mathrm{z}$ 818.51 to ionized o-TIPS with an attached third triisopropylsilanyl-ethynyl group, $\mathrm{C}_{22} \mathrm{H}_{11}\left(\mathrm{C}_{2} \mathrm{Si}(i-\mathrm{Pr})_{3}\right)_{3}{ }^{+}$. This ion may originate from a Diels-Alder reaction between the acene fragment of one o-TIPS and the alkyne fragment of a second o-TIPS molecule, followed by loss of the penta-
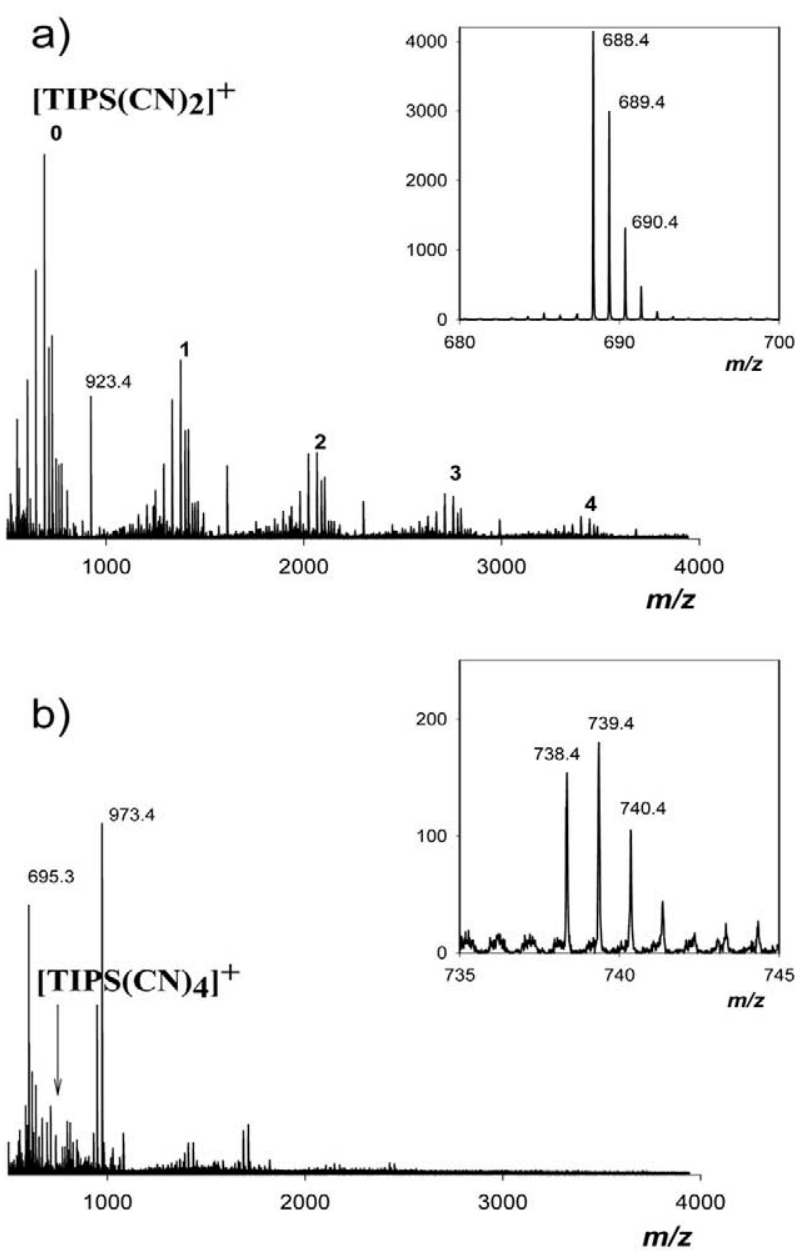

Figure 5. LDI spectra recorded for (a) TIPS $(\mathrm{CN})_{2}$ and (b) TIP$\mathrm{S}(\mathrm{CN})_{4}$ at a source pressure of $\sim 1$ torr with a declustering potential $(\mathrm{DP})=5 \mathrm{~V}$. (The intensity axis has a logarithmic scale). The inserts show the higher-resolution spectra for the monomer ions. The isotope pattern of the low-signal ions in the insert (b) can be accounted for by a mixture of TIPS $(\mathrm{CN})_{4}{ }^{+}$and $\operatorname{TIPS}(\mathrm{CN})_{4} \mathrm{H}^{+}$.

cene fragment of the added o-TIPS. The larger exposed $\pi$-face of o-TIPS makes this reaction only possible on this species, and not TIPS, TIPS $(\mathrm{CN})_{2}$, or $\operatorname{TIPS}(\mathrm{CN})_{4}$. It has been shown recently that the Diels-Alder reaction can take place in solution, provided there is a sufficiently reactive acene present [13]. However, the electrospray mass spectrum of the same sample taken independently with an API 4000 (AB/SCIEX) triple-quadrupole mass spectrometer did not show any of the ions at $\mathrm{m} / \mathrm{z} 818.51, \mathrm{~m} / \mathrm{z}$ 1456.89 , and $m / z 2095.25$ as contaminants. Therefore, we suggest that the third substituent in $\mathrm{C}_{22} \mathrm{H}_{11}\left(\mathrm{C}_{2} \mathrm{Si}(i-\mathrm{Pr})_{3}\right)_{3}{ }^{+}$ comes from neutral o-TIPS according to the ion-molecule reaction (eq 2) occurring in the laser plume.

$$
\begin{aligned}
& \mathrm{C}_{22} \mathrm{H}_{12}\left(\mathrm{C}_{2} \mathrm{Si}(i-\mathrm{Pr})_{3}\right)_{2}^{+} \\
& \quad+\mathrm{C}_{22} \mathrm{H}_{12}\left(\mathrm{C}_{2} \mathrm{Si}(i-\mathrm{Pr})_{3}\right)_{2} \rightarrow \mathrm{C}_{22} \mathrm{H}_{11}\left(\mathrm{C}_{2} \mathrm{Si}(i-\mathrm{Pr})_{3}\right)_{3}^{+} \\
& \quad+\mathrm{C}_{22} \mathrm{H}_{13} \mathrm{C}_{2} \mathrm{Si}(i-\mathrm{Pr})_{3}
\end{aligned}
$$

We note here that analogous reactions were not apparent with TIPS, TIPS $(\mathrm{CN})_{2}$, or TIPS $(\mathrm{CN})_{4}$. The mobility of 
the triisopropylsilanyl-ethynyl group described by eq 2, therefore, also appears to be restricted to o-TIPS. The $\mathrm{m} / \mathrm{z} 1456.89$ and $\mathrm{m} / \mathrm{z} 2095.25$ peaks can be identified as o-TIPS cluster ions of $\mathrm{m} / z$ 818.51, $\mathrm{C}_{22} \mathrm{H}_{11}\left(\mathrm{C}_{2} \mathrm{Si}(i-\right.$ $\left.\mathrm{Pr})_{3}\right)_{3}{ }^{+}(\mathrm{o}-\mathrm{TIPS})_{1,2}$.

TIPS $(\mathrm{CN})_{2}$ demonstrated trends toward clustering similar to those observed with TIPS (see Figure 5a). Cluster ions with up to four molecules of TIPS $(\mathrm{CN})_{2}$ $(\mathrm{m} / \mathrm{z} 1377.73,2066.1,2754.4$, and 3442.81) were observed and formed presumably according to the ion-molecule reaction (eq 1). Extensive fragmentation of the parent ion was observed resulting in $\mathrm{m} / \mathrm{z} 645.31,603.27,550.25$, and 522.25. These correspond to the neutral losses of $i \mathrm{Pr},\left[(i \mathrm{Pr})_{2}-\mathrm{H}\right], 2(i-\mathrm{PrCN})$, and $2(i \mathrm{PrCN})+\mathrm{C}_{2} \mathrm{H}_{4}$, respectively. The fragment ions also form clusters with neutral TIPS $(\mathrm{CN})_{2}$. Products of more complicated ion-molecule reactions with $\mathrm{m} / \mathrm{z}$ 727.32, 747.39 761.41, 775.45, 923.39 were observed, and these formed adduct ions with $\operatorname{TIPS}(\mathrm{CN})_{2}$.

TIPS $(\mathrm{CN})_{4}$ spectra did not demonstrate an intensive primary ion signal at $\mathrm{m} / \mathrm{z} 738.35$ (see Figure $5 b$ ). No clustering reactions appeared to occur although possibly the peak at $\mathrm{m} / \mathrm{z} 973.38$ is the adduct of $\mathrm{m} / \mathrm{z} 235$ that was not monitored. Comparatively abundant fragment ion peaks are evident at $\mathrm{m} / \mathrm{z} 713.35,638.37,603.10$, 587.10 , and 550.62, which correspond to multiple neutral losses of $\mathrm{CN}, i-\mathrm{Pr}$, and $\mathrm{C}_{2} \mathrm{H}_{4}$. The biggest peak at 603.10 can arise from the loss of the stable molecules $\mathrm{C}_{2}\left(\mathrm{CH}_{3}\right)_{4}+\mathrm{CH}_{3} \mathrm{CN}$. Attempts were made to reduce fragmentation and clustering through variation of the DP and laser fluence. Laser fluences of 50, 40, and 30 relative units were investigated as well as a DP equal to $0 \mathrm{~V}$, but these did not reduce the fragmentation of $\operatorname{TIPS}(\mathrm{CN})_{4}{ }^{+}$. So the laser fluence apparently is not critical under our experimental conditions and ionmolecule reactions dominate ion production in the laser plume.

\section{Discussion}

Collisional cooling is to be able to reduce the metastable fragmentation of analyte ion during the MALDI process $[5,6]$. There are two consecutive mechanisms of ion cooling that occur under the prOTOF 2000 experimental conditions: very rapid adiabatic expansion of the laserinduced plume into background gas and collisional relaxation in the RF only quadrupole ion guide. However, we have demonstrated (see Figures 3, 4, and 5) that in some instances the collisional cooling, even in combination with matrix, cannot completely quench fragmentation and promotes the ion-molecule reactions. The cluster formation, which should be considered as a manifestation of very successful cooling, could be suppressed by orthogonal means applying sufficient DP. On the other hand, additional drift potential leads to frictional heating of the ion population in strong collisional environments and so promotes fragmentation (declustering) as well as ion-molecule reactivity. TIPS $(\mathrm{CN})_{4}$ is a good example of a "problematic" mole- cule, which is just too fragile for direct desorption/ ionization, but highly reactive toward common matrix compounds.

In recent studies [5], two mechanisms were considered to account for cluster formation of analyte ions and matrix molecules and the effect of the DP. Briefly, according to one mechanism, the analyte ions emerge from hot clusters with matrix molecules that are formed in the desorption process. At 1 torr, these clusters could be "frozen" before evaporation is completed. The DP provides additional "heating" that can detach matrix molecules from the analyte ions. In the alternative mechanism the ions are formed and separated from matrix molecules at the early desorption stages in the plume. Ions, as well as matrix molecules, are "cooled" when the gas pressure in the MALDI chamber is high. Therefore, clustering is a product of gas-phase ionmolecule reactions between "cold" analyte ions and "cold" matrix molecules. Application of the declustering voltage should reduce the residence time of the ions in the plume and provide additional frictional heating preventing clustering. Careful experimental studies [6] have confirmed the second mechanism, which also provides a favorable background for the ion-molecule reactions.

The occurrence of extensive ion-molecule reactions indirectly confirms the second mechanism. We observed that sufficient frictional ion heating (DP above 10 $\mathrm{V}$ under our experimental conditions) largely prevents cluster formation, but concomitant reduction of the reaction time (residence time in the plume) does not have a significant effect on the extent of bimolecular ion-molecule reactions. One has to conclude that these complex reactions proceed at nearly the collision rate.

The pentacene ion-radical is expected to have the positive charge strongly delocalized along extensive $\pi$-orbital aromatic structure. We speculate that the pentacene clusters are created by electrostatic attraction of pentacene neutral molecule to positively charged pentacene ions creating sandwich-like structures with some inclination toward hydrogen atoms on the core of the molecular ion. The next pentacene molecule could be attached to the cluster similar to a solid-state herringbone ordering well known for pentacene [1].

The TIPS isomers possess bulky functional groups and the cluster formation is more complicated; the degree of the charge delocalization is expected to be even more intensive. An o-TIPS ion could directly interact with up to four aromatic rings of an o-TIPS molecule as presented in Figure $6 \mathrm{~b}$. Any other junction will be prevented by bulky functional groups from both sides. The same structure could be also considered as an intermediate in the ion-molecule reaction (eq 2). The interaction with the other side of the cation is sterically hindered, and contact through less than three rings apparently is not sufficiently strong under our experimental conditions (although the third cluster still can be observed in trace amounts). It is also possible that the positive charge is very effectively shielded in the first or 
second cluster, preventing following electrostatic interaction.

The highly symmetric TIPS molecule and ion at first glance should be even less predisposed to cluster formation than o-TIPS. TIPS has only two rings available for overlapping in a fashion similar to o-TIPS. Clustering should be suppressed by the steric hindering of the large functional groups. Our experimental observations (see Figure 4) demonstrate otherwise. We expect that the first TIPS cluster could be formed with a $60^{\circ}$ turn (see Figure 6a) around the center of the molecule. In this configuration, partial $\pi$-orbital overlap is still possible while keeping the functional groups sufficiently apart. Extensive cluster formation in the case of TIPS ions could be an indication that the positive charge is not sufficiently shielded in the first or second cluster, which corroborates the proposed structure.

The same cluster structure could be suggested for TIPS $(\mathrm{CN})_{2}$ ions. Two $\mathrm{CN}$ groups, attached to the pentacene carbons 2 and 3, take part in the charge delocalization and positioned in the aromatic plane. Such geometry does not assume significant steric hindrance to cluster formation. We speculate that if the TIPS $(\mathrm{CN})_{4}$ compound were more stable in the MALDI plume, it would make the same type of clusters as well.

One should not expect that the clusters created by ion-molecular reactions in the gas phase are similar to a fragment of the corresponding solid-state ordering. Thus, for example, the pentamer cluster (see Figure 6c) could be a logical choice [1]. Additionally, one could argue that the positive charge is highly delocalized and does influence the cluster structure. However, the orbital overlapping in this case is much weaker compared with Structure a in Figure 6.

\section{Conclusions}

Mass Spectrometric investigations of pentacene and its derivatives were performed using a novel MALDI TOF mass spectrometer with ion source normally at elevated pressure $\left(\sim 1\right.$ torr $\left.\mathrm{N}_{2}\right)$. Mechanisms of ion cluster formation of pentacene and functionalized pentacene compounds were discussed. A preferred model for cluster formation includes gas-phase ion-molecule reaction between "cold" analyte ions and neutral molecules. According to our speculation, pentacene can form clusters with structures similar to a fragment of the corresponding solid-state structure, while its functional derivatives create clusters specific to gas-phase electrostatic interaction. There now is a need for future more directed experiments with other pentacenes with different attached functional groups to provide further insight into these mechanisms.

It was determined that TCNQ assists the desorption of the intact pentacene and its derivatives by promoting the formation of radical cations. Although some ionmolecule reactions occurred, ion clustering of the compounds did not take place.

We also have determined that DHB and $\alpha$-CHC matrices, which are commonly employed in UV MALDI

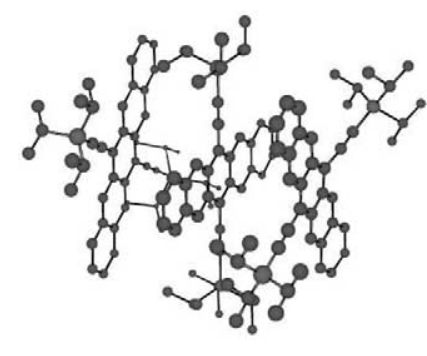

a)

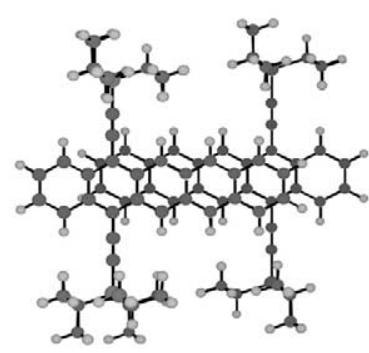

b)

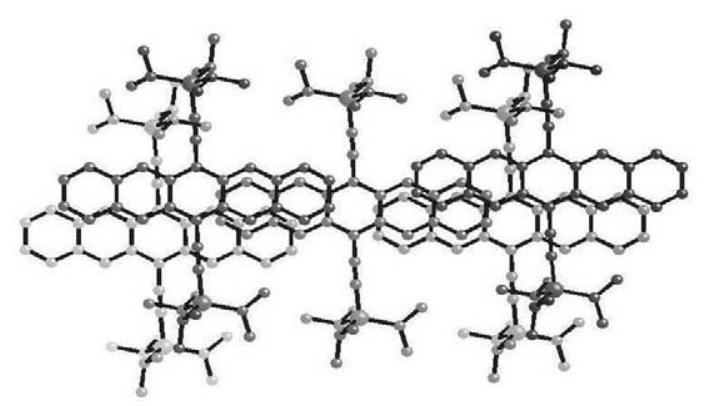

c)

Figure 6. Possible structures for gas-phase ion clusters of functional pentacene derivatives: (a) [TIPS] ${ }^{+}$(a spiral chain) and (b) [o-TIPS $]^{+}$. Structure (c) is proposed for a thin film of TIPS in the solid-state.

techniques to assist the desorption/ionization process, in this case are able to complicate significantly mass spectra via unwanted ion-molecule reactions of analyte ions with matrix molecules.

Very different results were obtained with direct laser desorption ionization of pure compounds. The difference in the pattern of cluster formation as well as the unique ion-molecule reactivity in the laser plume could assist, for example, in the analysis of two TIPS isomeric forms. For example, one of the observed ion molecular reactions of o-TIPS results in the attachment of a third functional group to the analyte ion, which is unique for this particular isomer. Two newly synthesized cyanic derivatives were investigated and the reactivities and stabilities of these compounds were investigated.

\section{Acknowledgments}

The authors acknowledge Dr. Suzanne Ackloo for her permission to use the MALDI-TOF instrument prOTOF 2000. They also gratefully acknowledge Dr. Peter Kovarik for his recommendations, technical support, and discussions of the Nebulizer Assisted-Continuous Track MALDI deposition technique, and Dr. Noemi Nagy for her permission to use the optical microscope system.

\section{References}

1. Anthony, J. E.; Brooks, J. S.; Eaton, D. L.; Parkin, S. R. Functionalized pentacene: Improved electronic properties from control of solid-state order. J. Am. Chem. Soc. 2001, 123, 9482-9483.

2. Ostroverkhova, O.; Cooke, D. G.; Shcherbyna, S.; Egerton, R. F.; Hegmann, F. A.; Tykwinski, R. R.; Anthony, J. E.. Bandlike transport in pentacene and functionalized pentacene thin films revealed by subpi- 
cosecond transient photoconductivity measurements. Phys. Rev. B 2005, 71, 035204-035206.

3. Hegmann, F. A.; Tykwinski, R. R.; Lui, K. P. H.; Bullock, J. E.; Anthony, J. E. Picosecond transient photoconductivity in functionalized pentacene molecular crystals probed by terahertz pulse spectroscopy. Phys. Rev. Lett. 2002, 89, 227403 (1-4).

4. Brooks, J. S.; Eaton, D. L.; Anthony, J. E.; Parkin, S. R.; Brill, J. W.; Sushko, Y. Electronic and optical properties of functionalized pentacene compounds in the solid state. Curr. Appl. Phys. 2001, 1, 301-306.

5. Loboda, A. V.; Ackloo, S.; Chernushevich, I. V. A high-performance matrix-assisted laser desorption/ionization orthogonal time-of-flight mass spectrometer with collisional cooling. Rapid Commun. Mass Spectrom. 2003, 17, 2508-2516.

6. Loboda, A.; Chernushevich, I. V. Investigation of the mechanism of matrix adduct formation in MALDI at elevated pressure. Int. J. Mass Spectrom. 2005, 240, 101-105.

7. Sundermeier, M.; Zapf, A.; Beller, M. A convenient procedure for the palladium-catalyzed cyanation of aryl halides. Angew. Chem. Int. Ed. Engl. 2003, 42, 1661-1664.

8. Swartz, C. R.; Parkin, S. R.; Bullock, J. E.; Anthony, J. E.; Mayer, A. C.;
Malliaras, G. G. Synthesis and characterization of electron-deficient pentacenes. Org. Lett. 2005, 7, 3163-3166.

9. Fung, K. Y.; Askovic, S.; Basile, F.; Duncan, M. W. A simple and inexpensive approach to interfacing high-performance liquid chromatography and matrix-assisted laser desorption/ionization-time of flightmass spectrometry. Proteomics 2004, 4, 3121-3127.

10. Wall, D. B.; Berger, S. J.; Finch, J. W.; Cohen, S. A.; Richardson, K.; Chapman, R.; Drabble, D.; Brown, J.; Gostick, D. Continuous sample deposition from reversed-phase liquid chromatography to tracks on a matrix-assisted laser desorption/ionization precoated target for the analysis of protein digests. Electrophoresis 2002, 23, 3193-3204.

11. Przybilla L.; Brand, J. D.; Yoshimura, K.; Rader, H. MALDI-TOF mass spectrometry of insoluble giant polycyclic aromatic hydrocarbons by a new method of sample preparation. Anal. Chem. 2000, 72, 45914597.

12. Gross, J. H; Mass Spectrometry, 4th ed.; Springer-Verlag: Berlin and Heidelberg, 2004, pp 417-418.

13. Payne, M. M.; Odom, S. A.; Parkin, S. R.; Anthony, J. E. Stable crystalline acenedithiophenes with up to seven linearly fused rings. Org. Lett. 2004, $6,3325-3328$. 